IRA-International Journal of Management \& Social Sciences

ISSN 2455-2267; Vol.04, Issue 03 (2016)

Pg. no. 590-601

Institute of Research Advances

http://research-advances.org/index.php/RAJMSS

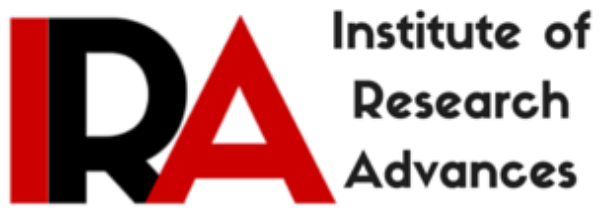

\title{
Industrial Clustering Leadership in Brownsville, Texas and Matamoros, Mexico
}

\author{
${ }^{1}$ Lewis Carrier $\&{ }^{2}$ Ify Diala \\ ${ }^{1,2}$ University of Phoenix, Phoenix AZ, USA.
}

Type of Review: Peer Reviewed.

DOI: http://dx.doi.org/10.21013/jmss.v4.n3.p9

\section{How to cite this paper:}

Carrier, L., \& Diala, I. (2016). Industrial Clustering Leadership in Brownsville, Texas and Matamoros, Mexico. IRA-International Journal of Management \& Social Sciences (ISSN 2455-2267), 4(3), 590-601. doi:http://dx.doi.org/10.21013/jmss.v4.n3.p9

(C) Institute of Research Advances

\section{(c)) EY-NC}

This work is licensed under a Creative Commons Attribution-Non Commercial 4.0 International License subject to proper citation to the publication source of the work.

Disclaimer: The scholarly papers as reviewed and published by the Institute of Research Advances (IRA) are the views and opinions of their respective authors and are not the views or opinions of the IRA. The IRA disclaims of any harm or loss caused due to the published content to any party. 


\begin{abstract}
The purpose of this case study was to understand the leadership forms and values that could affect organizational practices of an industrial cluster in the Brownsville/Matamoros region. A sample of 30 leaders from manufacturing companies in Brownsville, Texas, and Matamoras, Mexico participated in interviews and surveys. The analysis of the interview and survey data generated 12 major themes that emerged regarding the leadership forms, values, and cross-cultural challenges pertinent to the industrial cluster in the region. Participants viewed marked differences between American and Mexican leadership strategies, with emphasis on differences in procedural and power structures. Unifying goals and a commitment to learning about and understanding culture, family, and community may help foster respect and acceptance of cultural differences across the border. Cluster priorities for leadership include optimizing work conditions, education and training, resource utilization, and focusing on quality products and customer-oriented leadership. Planning, organization, and decentralized knowledge sharing, involving the combined knowledge, understanding, and experience of leaders, require communication, collaboration, and cross-functional teamwork. Education and training for current and future leaders and employees, with reasonable goals aligned with a unified vision for the cluster concept, encompasses measurable performance assessments based on goal achievement, supported by intrinsic and extrinsic rewards. The results from this study led to specific recommendations for leaders of the industrial sectors of Brownsville, Texas, and Matamoras, Mexico. The study concluded with limitations of the study and suggestions for future research based on the major thematic findings from this case study.
\end{abstract}

Keywords: Leadership, Industrial Cluster, Organizational practices, Knowledge Sharing

\title{
Introduction
}

Brownsville, Texas, and Matamoras, Mexico, are experiencing very high unemployment rates as a result of the loss of manufacturing jobs that left the area during the first decade of the $21^{\text {st }}$ century (CollReilly, 2011). Citizens of these two cities expressed concerned that the unemployment rates will continue to increase as more manufacturing companies relocate to China (Huang, Zhang, \& Liu, 2013). Several of the local political and industrial leaders discussed different methods to create jobs for these areas including the development of industrial clusters that would utilize existing competencies to produce new opportunities (Imagine, 2012).

Industrial clusters are geographically concentrated firms with similar technologies that usually consist of competitors, suppliers, distributors, and customers situated within close proximity to universities (Weng \& McElroy, 2011). These firms collaborate to create innovation to promote the growth of the existing members for their own economic self-interest. The firms involved in the industrial clusters develop vertical commonalities through buying and selling; they horizontally complement each other by the products and services they produce. All cluster members have something to offer that benefits the cluster and helps them to enhance their own potential growth. The local political leaders of Brownsville, Texas, and Matamoras, Mexico, realize that they must help the private sector make innovations in the marketplace in order to create new employment opportunities. One objective was to explore the concept of industrial clusters as a method of creating new jobs in Brownsville, Texas, and Matamoras, Mexico, However, the unique geography of the area may present leadership challenges that, if well understood, could be overcome. Overcoming leadership challenges that exist as a result of the cross-cultural nature of the border communities enhance the likelihood that an industrial cluster would be successful. The purpose of this descriptive case study was to understand the leadership forms and values that affect organizational practices of an industrial cluster in the Brownsville/Matamoros Borderplex region. 


\section{Purpose and Significance of the Study}

The purpose of this descriptive case study was to understand the leadership forms and values that affect organizational practices of an industrial cluster in the Brownsville/Matamoros Borderplex region. Leadership concepts are important in this study because, the success of any industrial cluster is dependent on the leadership that was developed specifically for the members of this cluster. The goals of this study included highlighting discoveries that contribute to understanding the leadership climate and challenges that affect the development and structure of an industrial cluster among the existing companies located in Brownsville, Texas, and Matamoras, Mexico. This qualitative study helps to fill a gap in the body of research left by previous attempts to study leadership concepts and the socio-economic complexities of industrial clusters. The process of research included a review of trends derived from peer-reviewed literature, personal interviews of experiences of the participants, and the use of a survey facilitating the methodological triangulation process that was integral to rigorous case study research (Yin, 2012). The creation of new manufacturing jobs from industrial clusters in Brownsville, Texas, and Matamoros, Mexico, might help to improve the economic conditions of this area. Raising a country's standard of living was dependent on the capacities of firms to achieve high levels of productivities by increasing productivities over time. Manufacturers face intense competition, uncertainty, and continuous change in $21^{\text {st }}$ century global markets; providing a product with high-quality at a low-cost does not guarantee success (Richardson, 2013). This study broadened the scope of research available to the organizations and political leaders of Brownsville, Texas, and Matamoras, Mexico, who are exploring alternative ways to create new jobs through industrial clusters. Understanding the necessary leadership required for a project of this magnitude was essential for the success of this venture. Organizational leaders and policy makers must understand the potential leadership challenges that affect industrial clusters; a driver for sustained regional development is the leadership process (Ferretti \& Parmentola, 2011) The study has significance for the governments, economic well-being, and citizens of Brownsville, Texas, and Matamoros, Mexico. The study was significant for the leaders of the area and for those involved in the development of new economic strategies as described below. The elaboration of the significance of the study pertains to both the significance to society and the significance to leadership.

Significance to society. The results of this research helped to identify information on the ideal ways to generate additional manufacturing jobs in Brownsville, Texas, and Matamoros, Mexico, by overcoming leadership challenges that exist as a result of the cross-cultural nature of this border community. The ideal ways evolved from an in-depth understanding of leadership issues that involved with or affected the industrial cluster. This information will be available to the City of Brownsville, Greater Brownsville Incentives Corporation, and the Brownsville Community Improvement Corporation so they can focus on the ideal approaches and overcoming challenges that affect leadership capacity when developing an industrial cluster.

\section{Discussions}

The following pages contain a review of the literature with a focus on leadership perspectives and the alternative method to create new manufacturing jobs in Brownsville, Texas, and Matamoros, Mexico, by developing an industrial cluster comprised from a strategic alliance of the existing companies. The deteriorating economies of the two geographical locations under study are great concerns for both communities. Business leaders, politicians, and educators from both sides of the border are seeking ways to create new jobs, including consideration of an industrial cluster by forming a strategic alliance between several of the existing companies on both sides of the border between the United States and Mexico.

Industrial Clusters. The term cluster has different meanings for different people; however, industrial clusters usually describe formal relationships between suppliers and buyers in the same geographical areas or with businesses that share production processes and technology in a joint effort to produce a standard product (Nishimura \& Okamuro, 2011). Industrial clusters develop from companies located in close proximity to each other; these companies work together to create productive and 
innovative environments to promote the growth, success, and productivity of existing members and create new approaches to strategic leadership and social responsibility (White, 2011). Cluster members share a common interest, mutually benefit each other, and are bound together solely by their economic selfinterest).

Holt (1998) described industrial clusters as tight networks of communicating, cooperating firms that drive innovation leading to prosperity from competition. Seyed and Ghanbari (2011) highlighted the collective efficiency of the industrial clustering mechanisms. Cluster organizations provide relative advantages compared to regional industries. Cluster organizations facilitate complementary relationships and can boost industry cohesiveness and exposure. Cluster organizations encourage collective identities and cooperative strength for improving regional prosperities. After the start of the $21^{\text {st }}$ century, the industrial clusters throughout the world exemplified growth in both quantity and quality of the products, variety, marketing, and management that they supply the world.

Industrial Cluster Leadership Considerations. Industrial clusters serve as employee attraction tools if leaders can positively reflect the economic importance of clusters to local industries. Local economic development organizations serve as leaders to assist companies trying to use cluster theory to expand the breadth and range of regional activities in particular fields. Having established that an automotive industrial cluster has the potential to include the Maquiladoras, it is important that leaders face the challenge of planning for and maintaining a positive reflection of competitiveness in a rapidly changing business environment along the U.S.-Mexican border. Leadership approaches should also create a foundation for identifying opportunities for collaboration, prioritizing strategies and actions, and documenting progress toward achieving cluster-specific goals, objectives, and outcomes. A firm's relationship between their leadership, manufacturing flexibilities, new product developments, and performance is essential if the firm is to stay competitive in new markets, especially within the automotive industry. Leadership teams are integral to manufacturing and production, but are also important to successful higher education initiatives, patent activity, and structuring of research-driven environments for the engineering, design, and business facets of automotive cluster plants.

Cross-Cultural Leadership Competence. Cross-cultural leadership competence involves sufficient knowledge about the skills, actions, and motivations of other leaders and followers that may function from the perspectives endorsed by, prevalent in, or specific to different nations (MirHosseini \& Ghanbari, 2011). Cultural uncertainty is a reflection of the abilities of team members to display patience, tolerance, and flexibility in multicultural and intercultural situations that may involve ambiguities and uncertainties stemming from cultural differences. Cultural empathy is the capacity to behave inquisitively with respect to others' cultures, display the communication patterns of others' cultures, and express a non-judgmental appreciation for the variety of working styles and the ways things are done in others' cultures.

Cross-cultural communication competence. Communicatively competent cross-cultural leaders are able to establish interpersonal relationships with foreign nationals through effective exchanges of verbal and non-verbal forms of communication behaviors. Muchiri and Cooksey (20011), argued that understanding the theoretical relationships between the national cultures and leaders' choices of effective communication strategies is necessary for effective exchanges and to generate a clear prediction about the role of effective leadership in a variety of contexts across cultures. Based on previous theoretical considerations of leadership effectiveness, five cultural orientations pertaining to communication include: (a) richness of the communications contexts; (b) power distances; (c) individualism-collectivism; (d) uncertainty avoidance; and (e) performance orientations. Communication contexts of cultures were described in terms of a continuum of low to high contexts. Low-context cultures emphasize explicit codes, words, and specific information-based messages. High-context cultures depend more on nonverbal contexts, physical settings, and the individuals' internalized values, beliefs, and norms surrounding 
the messages. Considering the proximity and cooperation among the two countries, there is a significant gap in the literature regarding cross-cultural study of the United States and Mexico.

Leadership-driven knowledge flow. Knowledge is considered a strategic resource for most organizations, which makes sharing it a key management concern. The success of a strategic alliance can depend heavily on effective knowledge exchange between the different companies. Sharing of human resources is typically influenced by the cost associated with the capital investments in the people to obtain specialized knowledge and skills; therefore, organizations may be hesitant to share knowledge or people with other organizations, but it is a requirement in a successful industrial cluster. Knowledge exchange in an industrial cluster depends on leadership and in an international cluster, may be affected by cultural differences. Cross-cultural leadership often relies upon knowledge facilitation by communication conventions, social exchanges, and common language among cultures. The concept of an industrial cluster relies heavily upon the exchange of ideas and transfer of knowledge.

Global Leadership and Organizational Behavior Effectiveness. The Global Leadership and Organizational Behavior Effectiveness (GLOBE) Research Program began in 1991 at the University of Pennsylvania. Two volumes of publications emerged between the start of the program and 2007 combined with several additional publications in peer-reviewed journals and research forums. The GLOBE research project began to measure the cultural differences among the various segments (countries, industries, and organizations) across the globe to explore leadership practices and values. The results were based on data from over 17,000 middle managers from almost 1,000 organizations involved with diverse service industries within 58 countries. GLOBE accounted for measures of culturally diverse ideas about the characteristics and attributes of effective leaders, thereby generating a large amount of data about cross-cultural leadership perspectives. GLOBE results were used to compare cultures in terms of their leadership values, practices, and styles endorsed in different cultures. Findings from the GLOBE surveys offer a range of practical data for cross-cultural businesses. GLOBE researchers concluded that some aspects of leadership are culturally dependent, while other leadership values, practices, and styles were more universally desirable. GLOBE established cultural dimensions that made it possible to capture the similarities and differences in leadership norms, values, beliefs, and practices among societies with different cultural orientations. Specific focus was placed on transformational and charismatic leadership styles, also called value-based leadership dimensions; charismatic style characteristics contributed more than other dimensions to leadership styles that were perceived as more universally effective (House et al., 2013). The GLOBE findings were also discussed and used in subsequent research about bicultural leaders. For example, Brannen and Thomas (2011) examined the implications associated with the idea that cross-cultural leadership research typically assumed that individuals had only one cultural profile. In light of the changing patterns of a globally-connected workforce, Brannen and Thomas (2011) highlighted the increasing trend for more employees, managers, and leaders being or working within bicultural individuals. The author underscored the need to further understand the emerging bicultural demographics of organizations. Thomas, Brannen, and Garcia (2011) defined biculturalism as people who internalized more than one cultural profile. The authors claimed that biculturalism is the result of globalization and represent an emerging, growing demographic that present important challenges and opportunities for international leadership. Thomas et al. (2011) demonstrated that biculturalism fosters the general skills that can be beneficial to multinational organizations; study results indicated that biculturalism offers a pronounced skill set related to intercultural effectiveness that included higher levels of cognitive skills called cultural metacognition that directly impacts intercultural effectiveness.

\section{Methodology}

This qualitative, descriptive-case study focused on the leadership forms and values that affect organizational practices of an industrial cluster in the Brownsville/Matamoros Borderplex region. Population for this study included the Matamoros Maquiladora Association, also known as AMMAC (Asociación de Maquiladoras de Matamoros A.C.), formed in 1972 and the Brownsville Economic 
Development Council (BEDC) is a business organization that promotes the economic and industrial development in Brownsville, Texas, and Matamoros, Mexico. The BEDC was publicly-funded in 1992. The specific sampling criteria includes that each participant must be a member of the BEDC or AMMAC, and must be knowledgeable with the concepts pertaining to industrial clusters, strategic alliances, and leadership values, styles, customs, and strategies as they pertain to this specific geographical area. Participants were willing to participate and sign an informed consent form in order to participate. Participants were all fluent in the English language, although their first language can be English, Spanish, or another language. Every participant was interviewed by the researcher so all prospective participants were willing to participate in a personal interview. This study consisted of 30 participants who were all leaders in the manufacturing industries in Brownsville, Texas, and Matamoras, Mexico. All of these leaders were members of the Canacintra de Matamoras, Mexico, Brownsville Economic Development Council and/or the Matamoros Maquiladora Association. The responses of 30 participants were analyzed with the help from qualitative research software Nvivo 10. The results of the Nvivo 10 program indicated that there were 12 themes identified from the surveys and interview data. The BEDC and the AMMAC maintain public websites with the names, emails, and telephone numbers of members. The contact information was publically availableThe data collection method of the current study interviewed individuals using an open-ended questioning strategy combined with a survey that was determined to be the best data collection and triangulation method for this study. This study involved a field test, personal interviews, and a survey. The participants of this study were all adult, English-speaking leaders and members of the Brownsville Economic Development Council and the Matamoros Maquiladora Association. Descriptive case study research using triangulated data, such as focus groups, interviews, surveys, and secondary data, can synthesize information from participants involved with a case about their experiences and perceptions on a variety of topics (Throupe, 2011). The information gathered from the participants in a variety of ways was used to triangulate data in order to make good judgments and support those judgments for valuation decisions (Shoemaker, Kazley, \& White, 2011). A purposive sample of the population was appropriate for this study because the participants were selected by the researcher based on specific criteria. Purposive sampling is a form of non-probability sampling whereby decisions to include individuals in the sample are made by the researcher, based on specific criteria that includes knowledge of the research issues and the willingness to participate in the research.

\section{Data Analysis}

Data analysis occurred by qualitative content analysis. Priest, Roberts, and Woods (2011) suggested that content analysis is a reliable method for analyzing qualitative data. The integration of qualitative content analysis as a method of examination of data in case study research. Bendersky and Kathleen (2011) stated that contemporary content analysis is a method that can offer an exploratory process with predictive intents. Qualitative content analysis involves searching the participants' transcripts to locate themes and patterns to comprise meaningful units that consist of small bits of text that are independently able to convey a specific meaning. After the data was collected, data was categorized according to meanings generated from the sample. This method included a process that listed and categorized statements from the interviews to determine value. The data was then analyzed with focus on detailed narratives that explore patterns of the responses from the interviews. The patterns were then used to identify specific themes that were necessary to address the research questions qualitative content analysis results in unique themes that illustrate the range of the meanings of particular texts or concepts (Vaismoradi et al., 2013). The first step in the qualitative content analysis was preparing the data. The narrative data from interviews were transformed into written text before analysis starts. When transcribing interviews, all the questions of the interviewer were transcribed and verbalizations were transcribed literally. The second step was defining the unit of analysis; a theme could be a single word, phrase, sentence, or paragraph. When using themes as the coding unit, the researcher was primarily focused on the expressions of an idea. The third step involves developing categories and coding schemes derived from three sources: the data, previous published relative studies, and conceptual theories. The constant comparative method allows for systematic comparisons of each text, the integration of categorical 
properties through the reliance upon interpretive memos and the development of coding notes, and the checking of coding consistencies. Categorical coding and constant comparisons lead to the identification of relationships between categories, revealing patterns, and themes important to the social reality. The process of qualitative content analysis was supported by the computer aided qualitative data analysis software program, NVivo 10. The program assists researchers as they organize, manage, and code qualitative data, helping the process occur in a more efficient manner. The program supports text editing, coding, text retrieval, categorical and node manipulation. Visual presentation modules allow the researcher to more vividly see the relationships between categories. The data collected from surveys was inexpensive, convenient, easily gathered, easily tabulated, and the researcher extrapolated attributes of a population from a small sample on a computer. After the data was collected from the personal interviews, the next step included participant completion of the GLOBE survey followed by data entry of the responses into Excel. The survey results were compiled into descriptive reports, with comparisons between surveys from respondents in the United States and Mexico to each other and to the qualitative narrative interview data. The comparisons reflected the frequencies of responses, means, median, mode, and standard deviations of and between respondents, can provide more detail and provide for areas of convergence and divergence among participant reports. The combination of the survey analysis with the interview data analysis provided a more meaningful set of data. After transcription of the interview notes, a process occurred involving reading and re-reading data with the purpose of developing an appreciation for the overall concepts expressed by participants. Note taking about potentially important words, patterns, and phrases happened throughout the initial data review process. Textual data then underwent a computerized data analysis process involving the identification of categories of key words and phrases and that were prominent in the data. Frequency counts as well as coding involving the identification of categories of words and terms emerged from the computerized data analysis process. Grouping of like terms and phrases led to the identification of additional patterns in the data. The report of the data analysis of the interview data, including results from computerized coding processes involving those key terms, words, phrases, and categories follow.

\section{Findings and Conclusions}

Twelve themes emerged from the analysis of the data combining the findings from both the interviews and survey answers. Participants' quotes provide relevant examples that provide context and additional support for the major thematic findings. The themes pertained to the priorities of the cluster that would involve education and training, resource utilization, and maximizing profits. Planning, organization, and decentralized knowledge sharing should involve leadership, communication, collaboration, and support. Cross-functional teamwork and open communication through frequent meetings are essential to the success of the cluster. Leadership behaviors, education, and training were key aspects of the data leading to related themes. Leaders of the cluster will need to establish clear reasonable goals aligned with a unified vision for the cluster concept, based on their combined knowledge, understanding, and experience. Motivators were also important aspects of the data leading to thematic findings. Differences between American and Mexican leadership strategies, as well as ways foster respect and acceptance of cultural differences across the border, emerged from the data.

\section{Theme 1: The development of a manufacturing cluster would bring more jobs to the sectors of Brownsville, Texas, and Matamoras, Mexico, that could help vitalize the areas.}

The results of the study indicated that participants believed in the potential of the industrial cluster as a source of new jobs that could help to reduce unemployment in the area. Raising a country's standard of living depends on the capacities of firms to achieve high levels of productivities by increasing productivities over time. According to participants in the study, the standard of living in the area is one major perceived advantage of a new industrial cluster that can sustain productivity over time. The perceptions of such a driver for sustained regional development that would depend on effective leadership processes were consistent with the findings of the study. 
Theme 2: Human resources must focus on work conditions, including pay, benefits, and attention to education, health, and safety issues.

The conclusions were that important issues for success are attention to internal and external relationships, including the types of inter-organizational and governmental relationships that can work in tandem to ensure optimal working conditions for employees. The participants emphasized the potential benefits of the cluster to the overall infrastructure, economy, affiliated companies, institutions, and communities, should be important agendas for governments and associated stakeholders of the involved cluster communities. Comparison to the United States, Mexico can fall short of providing good opportunities for education, training, and work incentives. The importance of human capital to sophisticated automotive cluster activities, with emphasis assessing and addressing specific work conditions, increasing education and skills development, and adopting high leadership standards. Participants in the study emphasized the potential positive benefits of addressing the social, education, and vocational needs of the Borderplex region workforce.

Theme 3: Priorities of the Matamoros/Brownsville cluster would involve education and training, resource utilization, and maximizing profits.

Consistent with the participants' emphasis on resource utilization was the discussion that could enhance industrial cluster competitiveness, increasing productivity by encouraging member specialization in technology, information, and specific resources resulting in unique utilization capabilities that can lead to increased profitability. Individual cluster members also improve their designs and innovations through the development of strategic alliances. According to participants, such strategic alliances help reduce costs and improve innovation and technology leading to quality improvements, consistent with the strategic alliance outcomes of organizations. Such alliances, from the viewpoints of participants would, require attention to education, training, and high levels of technology for communication and collaboration leading to optimal productivity, efficiency, and worker motivation.

Theme 4: Success of the cluster depends on quality products and customer-oriented leadership based on feedback and good relations among the stakeholders of the business.

Results of the study indicated that emphasis on cost in light of quality should be a priority. However, providing a product with high-quality at a low-cost does not guarantee success. Study participants did emphasize high quality and low cost, but did not attribute the potential success of the cluster exclusively to those two necessary facets. Equally important to a leader's ability to maintain automotive cluster cost structure, level of quality, and delivery. performance are relationships, which were concepts the participants also discussed as important.

Study participants discussed strategic alliances in ways that indicated the alliances could improve education, training, and resource utilization, leading to higher profits for the company and economic benefits for the communities; efficiency was a concept important to participants in the study as was responding to the needs and expectations of customers and key stakeholders. Participants described leadership as leaders who get to know their environment, are proactive, foresee opportunities, and develop collaborative resource sharing to pursue opportunities with stakeholders of the organization to produce desired results.

Theme 5: Planning, organization, and decentralized knowledge sharing involving leadership, communication, collaboration, and support are essential to a successful cluster strategy

The importance of the early phases of industrial cluster development in the automotive industry and how research can help initiators overcome the collective action problems of creating a cluster group. The majority of participants in the study claimed that planning was one of the most important aspects that needed strong leadership to realize cluster success. The results of the study indicated that strong leadership throughout the planning, implementation, and operating phases is essential to the success of the cluster. Participants stressed inter-organizational coordination as well as intra-organizational coordination and regular collaboration to help participating organizations to pursue competitive positions in the global 
market, Collaboration and coordination were among the major concepts that emerged from the data that participants claimed were essential to the success of the cluster. Results of the study indicated that knowledge exchange was a key concept emphasized by participants in the study, the importance of knowledge sharing as a key factor of success in most of the cases studied. Although only five participants highlighted the potential outcomes of leadership as empowerment, the majority did emphasize the importance of knowledge sharing and communications, claiming that it would help with efficiency, productivity, and collaboration.

Theme 6. Promoting cross-functional teamwork and open communication through frequent meetings are essential to the success of the cluster

The results of the study indicated that leadership focused on collaboration, communication, and team building could best support the cluster. Participants spent time each discussing the importance of the contributions of employees to the leadership and management process. They discussed ways that leaders could manage discomfort and anxieties productively, Leaders who are adaptive develop interdependent interactions through the sharing of ideas, information, and resources that are not reflective of any one contributor which participants in the study claimed would be beneficial to the cluster process. The participants in the study appeared to focus on team effectiveness more than the others. Although, they did discuss extensive cultural competencies, uncertainties, and empathy as well as interpersonal skills, the idea of cluster success stemmed more from focus on team building as a primary focus with the ideas related to cultural competencies as a component of those team processes. The results of the study that indicated strong recommendations for collaboration and team building are consistent with the prior literature that indicated these strategies could lead to competitive advantages (Nishimura \& Okamuro, 2011).

Theme 7 Leadership behaviors deemed appropriate for the cluster include being involved and leading by example, establishing trust and dedication, and showing the sincerity, respect, charisma, and inspiration to effectively motivate followers.

There were marked differences between several Globe survey scale item means of the U.S., Mexico, and the participants in the study. The sample means for charismatic/value based styles were higher than means from Mexico but lower than means from the U.S. The sample means were higher than world means for all of the characteristics except integrity, decisive, and self-sacrificial. Analyses conducted of cultural dimension scales showed how different leaders agreed and in terms of other leadership styles applied cross-culturally in this study, prior research indicated that transformational leadership had positive effects on core quality management practices and essential infrastructures; transactional leadership strategies had less positive influence on the same factors. Participants discussed leadership in terms of inspiration and motivation as well as managerial tactics for efficiency, but emphasized the integration of management and leadership. Participants considered establishing trust between the leaders and the followers as more essential. Findings from the study were that the participants appeared less concerned with the actual types of labeled leadership behaviors as they were with how those behaviors ultimately materialized into effective practices. For example, group cohesion, leader-member relationships, employee satisfaction, productivity, and growth toward the collective goals were prominent concepts in the data, with discussions of how to bring about positive outcomes in those areas, as opposed to talk about specifically labeled leadership behaviors.

Findings of the study also showed that high charismatic and value based leadership ratings by participants that were the dimensions that contained the largest number of attributes universally perceived by research participants in different studies in different countries as contributors to effective leadership.

Theme 8 Education and training should encompass leadership training for current and future leaders, oriented toward a vision and understanding of the cluster concept.

The results of the study indicated that the participants were familiar with the leadership traits known to be successful in business practices, with some standing out as more desirable than others in the 
industrial cluster process. Results of the study indicated that participants felt that cultivating the skills and talents of current and future leaders is possible through leadership training, and represents important components of the cluster process. Leaders preparing leaders was a concept stressed in the data collected and participants in the study stressed learning, knowledge, and understanding about both leadership and the cluster process as requirements for a successful cluster process. Problems with organizational leadership that involve cross-cultural or intercultural. Participants in the study emphasized leadership development and diversity training for both leaders and followers.

Theme 9 Leaders of the cluster will need to establish clear reasonable goals aligned with a unified vision for the cluster concept, based on their combined knowledge, understanding, and experience

Cooperative goals, will enhance the personal meaningfulness and importance of a member's willingness to fulfill the visions communicated by leaders. The commitment of a shared vision brings unity within an organization and brings members closer together, which were insights also shared by participants in the study. Participants highlighted the combination of a strategic managerial leader and a visionary leader, which is what participants appeared to emphasize as necessary for the cluster leadership. Strategic leadership involves setting specific targets and time frames for the organization to follow, which were included among the recommendations from participants in the study. key players in the automotive industry can learn each other's experiences and adopt good general development and management strategies based on the documented experiences of other clusters. Participants in the study suggested that the strength of the cluster will stem from the combined knowledge, understanding, and experience of the leaders involved participants in the study indicated that knowledge and experience from many different sources strengthens business strategies and a strategic alliance that crosses international borders requires all of the partners to incorporate cognitive diversity to understand the different organizational cultures of their partners.

Theme 10 Formal, measurable performance assessments based on goal achievement should lead to intrinsic and extrinsic motivational rewards

Performance orientation pertains to rewards and motivations toward improvements in light of the concepts of excellence; low performance-oriented cultures value traditions, loyalties, and families, while high performance-oriented cultures strongly emphasize performance value, training programs, professional development, and advancement incentive (MirHosseini \& Ghanbari, 2011). According to participants, the prospective workforce values traditions, loyalties, and families but also should be encouraged with performance-oriented incentives. Transactional leaders focus on supervision, organizational roles, and group performance, thereby promoting compliance of followers through rewards and punishments (Ceri-Booms, 2011), which were aspects of the suggestions that participants made for the success of the industrial cluster. According to Ceri-Booms (2011), the main concern for these leaders is to meet or exceed the organizational goals and objectives by giving contingent rewards to their employees; these leaders gain commitment from employees by contingent rewards. Transactional leaders maintain consistent rules, procedures, and maintain individual goals, and rewards (Arnold \& Loughlin, 2013) that participants also claimed would be important to the success of the industrial cluster. While rewards structured toward performance and goals were prominent aspects of the data collected, transactional leadership does not inspire the same shared vision, trust, loyalty, or admiration of the followers as participants claimed would be necessary for effective cluster leadership.

Theme 11 Participants viewed marked differences between American and Mexican leadership strategies, with emphasis on differences in procedural and power structures.

There may be unique considerations in a cluster industry that crosses cultural, societal, economic, and physical borders. The participants offered insight into the potential differences of leaders of the cluster process. While some participants did not expect significant differences in leadership behavior 
between American and Mexican leaders primarily due to proximity across the border, others did note the potential for cultural, societal, and even language differences.

Mexico was characterized as relatively substandard in productivity, education, and innovation with lower wages, poorly effective unions and corruption and crime as significant barriers to conducting business in Mexico. Participants in the study brought up all of these concerns and discussed perceptions about procedural and power structures that could differ between the U.S. and Mexico. Based on previous theoretical considerations of leadership effectiveness, dimensions used to illustrate the embedded values of different cultures included power distance, uncertainty, masculinity and femininity, individualism and collectivism all of which were expressed by various participants in the study as representing potential differences among U.S. and Mexican leadership practices and expectations.

\section{Theme 12 Unifying goals and a commitment to learning about and understanding culture,} family, and community will help foster respect and acceptance of cultural differences across the border.

Participants in the study verbalized the ideas that cross-cultural leadership competence involves sufficient knowledge about the skills, actions, and motivations of other leaders and followers that may function from the perspectives endorsed by, prevalent in, or specific to different nations. the majority of participants in the study did not appear to be greatly concerned with how to resolve. Thomas et al. (2011) and Hong (2011) discussed biculturalism as people with two internalized cultural schemas otherwise known as the set of knowledge about and experiences pertaining to the values, norms, and beliefs of a specific culture. The majority of participants in the study could be characterized as bicultural, based on their reported demographic data and experiences, but the idea was expressed that exhibiting bicultural competence can be learned to maximize members' contributions in ways that positively impact multicultural team effectiveness.

\section{References}

Arnold, K. A., \& Loughlin, C. (2013). Integrating transformational and participative versus directive leadership theories. Leadership \& Organization Development Journal, 34(1), 67-84. doi:http://dx.doi.org/10.1108/01437731311289974

Bendersky, C., \& Kathleen, L. M. (2011). Open to negotiation: Phenomenological assumptions and knowledge dissemination. Organization Science, 21(3), 781-800. Retrieved from http://search.proquest.com

Brannen, M. Y., \& Thomas, D. C. (2011). Bicultural individuals in organizations: Implications and opportunity. International Journal of Cross-Cultural Management, 10(1), 5-16

Ceri-Booms, M. (2011). An empirical study on transactional and authentic leaders: Exploring the mediating role of trust in leader on organizational identification. The Business Review, Cambridge, 14(2), 235-243. doi: 34756548735812

Coll-Reilly, J. (2011). Relocating millions: The Chinese answer to development. The International Business \& Economics Research Journal, 9(11), 143-147. Retrieved from http://search.proquest.com/docview/818455860?accountid=35812

Ferretti, M., \& Parmentola, A. (2011). FDI knowledge spillovers and host government policies: The Iranian experience. European Business Review, 22(2), 175-194. doi:http://dx.doi.org/10.1108/09555341011023515

Hong, H. J. (2011). Bicultural competence and its impact on team effectiveness. International Journal of Cross-Cultural Management, 10(1), 93-120

House, R., Dorfman, P., Javidan, M., Hanges, P., \& Sully de Luque, M. (2013). GLOBE study of CEO leadership behavior and effectiveness in 24 countries. Thousand Oaks, CA: Sage

Huang, G. Q., Zhang, A., \& Liu, X. (2013). A supply chain configuration model for reassessing global manufacturing in china. Journal of Manufacturing Technology Management, 24(5), 669-687. doi:http://dx.doi.org/10.1108/17410381311327963

Imagine (2012). Brownsville. Retrieved from http://www.imaginebrownsville. 
com/home.php.

MirHosseini, S. V., \& Ghanbari, M. R. (2011). Investigation of the industrial clusters in Islamic Republic of Iran (case study of Yazd city using local production systems method \& industry perception model). International Journal of Academic Research in Business and Social Sciences, 1(3), 357366. Retrieved from http://search.proquest.com/docview/1027115638?accountid=35812

Muchiri, M. K., \& Cooksey, R. W. (2011). Examining the effects of substitutes for leadership on performance outcomes. Leadership \& Organization Development Journal, 32(8), 817-836. doi:http://dx.doi.org/10.1108/01437731111183757

Nishimura, J., \& Okamuro, H. (2011). R\&D productivity and the organization of cluster policy: An empirical evaluation of the industrial cluster project in Japan. Journal of Technology Transfer, 36(2), 117-144. doi:http://dx.doi.org/10.1007/s10961-009-9148-9

Priest, H., Roberts, P., \& Woods, L. (20010). An overview of the three different approaches to the interpretation of qualitative data. Part 1: Theoretical issues. Nurse Researcher, 10(1), 30-43. Retrieved from EBSCOhost.

Richardson, C. (2013). Knowledge-sharing through social interaction in a policy-driven industrial cluster. Journal of Entrepreneurship and Public Policy, 2(2), 160-177. doi:http://dx.doi.org/10.1108/JEPP-08-2011-0010

Seyed-Valiollah, M. H., \& Ghanbari, M. R. (2011). Investigation and analysis of the performance of industrial clusters in Islamic Republic of Iran. International Journal of Business and Social Science, 2(15), 14-23. Retrieved from http://search.proquest.com/docview/904521797?accountid=35812

Shoemaker, L., Kazley, A., \& White, A. (2011). Making the case for evidence-based design in healthcare: A descriptive case study of organizational decision making. HERD: Health Environments Research \& Design Journal, 4(1), 56-88. Retrieved from http://search.proquest.com/docview/856214613?accountid=35812

Thomas, D. C., Brannen, M. Y., \& Garcia, D. (2011). Bicultural individuals and intercultural effectiveness. European Journal of Cross-Cultural Competence and Management, 1(4), 315-333

Throupe, R. (2011). The use of focus groups for property valuation research. The Appraisal Journal, 79(4), 301-313. doi:91525888735812

White, T. (2011). A new approach to business ethics: Strategic response, leadership and social responsibility. The IMS Journal of International Business, 1(1),

Yin, R. K. (2012). Qualitative study research: Design and methods (4th ed.). Thousand Oaks, CA: Sage. 